Revue d'histoire du XIXe siècle

Société d'histoire de la révolution de 1848 et des

révolutions du XIXe siècle

Religion, politique et culture au XIXe siècle

Michel Arrous [dir.], Alexandre Dumas, une lecture de l'histoire, Éditions Maisonneuve et Larose, 2003, $617 \mathrm{p}$.

Judith Lyon-Caen

\title{
OpenEdition
}

Journals

Édition électronique

URL : http://journals.openedition.org/rh19/643

DOI : 10.4000/rh19.643

ISSN : $1777-5329$

Éditeur

La Société de 1848

Édition imprimée

Date de publication : 1 juin 2004

Pagination : 201-203

ISSN : 1265-1354

Référence électronique

Judith Lyon-Caen, « Michel Arrous [dir.], Alexandre Dumas, une lecture de l'histoire, Éditions Maisonneuve et Larose, 2003, 617 p. », Revue d'histoire du XIXe siècle [En ligne], 28 | 2004, mis en ligne le 21 juin 2005, consulté le 22 septembre 2020. URL : http://journals.openedition.org/rh19/643 ; DOI : https:// doi.org/10.4000/rh19.643

Ce document a été généré automatiquement le 22 septembre 2020

Tous droits réservés 


\section{Michel Arrous [dir.], Alexandre Dumas, une lecture de l'histoire, Éditions Maisonneuve et Larose, 2003, 617 p.}

Judith Lyon-Caen

Issu du colloque Dumas qui s'était tenu à la Sorbonne le 16, 17 et 18 octobre 2002 à l'occasion du bicentenaire de la naissance de l'écrivain, cet ouvrage collectif propose une réévaluation des relations de Dumas et de l'histoire. Il s'agit par-là d'examiner non seulement les époques de prédilection de Dumas, mais aussi ses méthodes de travail ainsi que ses choix et ses usages des sources historiques; de s'interroger sur la portée historique de la fiction dumasienne à une époque où le savoir historique en train de se constituer s'intéresse aux ressources du récit et de la fiction; de réfléchir, enfin, à l'écriture d'une histoire "du temps présent», depuis les romans du "cycle révolutionnaire " (Joseph Balsamo, Le Collier de la Reine, Ange Pitou, La Comtesse de Charny) jusqu'aux Mohicans de Paris, en passant par Le Comte de Monte-Cristo et les évocations multiples de la figure napoléonienne. Cet ouvrage réunit 31 contributions, que les dimensions de cette notice ne permettent pas d'étudier successivement. Nous suggérerons seulement un parcours dans ce riche ensemble, au sein duquel trois types de contributions peuvent être distingués: les premières rendent compte du fonctionnement de l'écriture de Dumas en général, du rapport de ses fictions avec le passé et avec les autres genres historiques (la chronique, l'histoire); les deuxièmes esquissent une réflexion sur le rapport de Dumas à l'histoire de son propre temps, par le biais notamment d'un questionnement sur la signification politique de certaines œuvres; les troisièmes s'intéressent aux différents terrains dumasiens, espaces géographiques comme l'Italie ou la Grèce ou moments historiques privilégiés.

Charles Grivel et Sarah Mombert posent la question, centrale, du rapport de la fiction et de l'Histoire. Le premier l'aborde dans une perspective théorique, en évoquant d'ailleurs quelques réjouissants morceaux de théorie du roman enchâssés dans l'œuvre 
de Dumas. Estimant que seule la narration permet de montrer toutes les facettes d'un objet historique, Dumas propose une recomposition narrative de l'histoire, qui, s'appuyant sur des destins individuels et des événements singuliers, libère une écriture indissociablement historique et fictionnelle. Le texte dumasien se construit à partir de tous les matériaux écrits disponibles à l'écrivain (livres anciens ou manuscrits), qu'il capte et récrit pour produire un récit pertinent. Les règles de cette écriture renvoient, selon Charles Grivel, à deux attentes fondamentales du lecteur : «celui d'être informé sur ce qui est et celui d'être renseigné sur ce qui n'est pas avéré ». Double désir, de savoir et d'imaginaire, qui est au principe du succès de l'entreprise de vulgarisation dumasienne, dont Sarah Mombert examine la genèse, les ressorts et la portée. Dumas s'est voulu vulgarisateur, et l'a incontestablement été, en donnant le goût de l'histoire à des générations de lecteurs. Son entreprise a d'ailleurs si bien réussi, note Matthieu Lettoumeux dans une contribution consacrée à la descendance européenne de Dumas, que le roman de "cape et d'épée " à la manière de Paul Féval ou de ses homologues ne se déroule pas dans la France "du passé » mais dans la France de Dumas. Dumas a d'abord investi le drame historique, puis la chronique et enfin le roman, qu'il avait d'abord jugé impropre à rendre l'histoire vivante. Il ne parvient, souligne Sarah Mombert, à trouver un compromis entre savoir historique et roman qu'en 1844, avec Les Trois Mousquetaires. Comment s'y prend-il ? En adoptant le point de vue du roman de mœurs de l'époque, celui des envers de la grande histoire, de la vie privée, des secrets intimes. C'est ce point de vue à la fois particulier et universel qui permet à Dumas de gagner à l'histoire le nouveau public du roman, le public populaire et féminin (et Dumas, comme Balzac, s'est souvent complu à évoquer sa complicité avec ses «belles lectrices»). Le romancier voyait son œuvre comme un vaste programme d'éducation populaire à visée émancipatrice : son entreprise aurait ainsi réalisé l'idéal politique qu'il n'a jamais pu incarner dans l'action, durant sa brève aventure politique sous la II ${ }^{\mathrm{e}}$ République.

3 La question du sens politique de l'œuvre de Dumas traverse toutes les contributions qui s'intéressent, sous différents angles, au rapport de l'écrivain au contemporain. Dans son théâtre, tout d'abord, étudié ici par Anne Ubersfeld qui suggère combien l'univers parlementaire de la Monarchie de Juillet constitue non seulement le cadre implicite, mais souvent le sujet, de pièces comme Angèle ou Richard Darlington. Face aux héros de Dumas, monstres d'ambition et d'orgueil, hommes de la démesure, le monde social apparaît sous les couleurs sombres du cynisme et de la brutalité. Les nombreuses contributions consacrées dans ce volume au Comte de Monte-Cristo et, surtout, aux romans du cycle révolutionnaire suggèrent également l'importance du questionnement de Dumas sur la mort politique, la violence et l'injustice (Sylvain Ledda, Claudie Bernard). Dumas a été habité par le souci de penser l'histoire, de lui donner une cohérence et un sens : d'où son travail sur des personnages historiques controversés ou flous, comme Richelieu et Louis XVI, et sa prédilection pour la période révolutionnaire (Jean Tulard, Laurent Giraud). Denis Saillard revient ici sur les romans de la fin de la vie du romancier, comme la San Felice où, en évoquant la République napolitaine de 1799, Dumas réfléchit sur la possibilité d'une République exigeante qui ne dérive pas dans la violence et la Terreur. Le propos politique est donc constant, mais il n'est pas toujours lisible, polyphonie romanesque oblige. Anne-Marie Callet-Bianco peut néanmoins retracer l'affirmation du sentiment républicain entre Le comte de Monte-Cristo (1844) et Les mohicans de Paris (1854). Jean-Yves Mollier aborde également la question politique en revenant sur l'épisode de la candidature de Dumas aux élections d'avril 1848 : Dumas 
s'était présenté devant les électeurs de Seine-et-Oise en retournant à son profit le discours sur "l'industrialisation» de la littérature pour se dépeindre sous les traits d'un travailleur infatigable (qu'il était), entrepreneur en écriture capable de fournir du travail à des centaines d'ouvriers imprimeurs, papetiers ou lithographes. Porte-parole d'un républicanisme modéré, il formule alors une passionnante réflexion sur l'identité économique et sociale de l'homme de lettres.

Le rapport de l'écrivain au contemporain est également au cœur de toutes les contributions qui abordent la question du rapport de Dumas avec la science historique de son temps. Il est difficile de saisir le statut de l'écriture de Dumas si l'on pétrifie trop les frontières, encore incertaines, du savoir historique et de l'écriture littéraire : Dumas avait été émerveillé par la vie qui émanait des Récits sur les temps mérovingiens d'Augustin Thierry ; son Moyen Âge est tout imprégné de Scott, bien sûr, mais aussi des historiens romantiques (Isabelle Durand-Le Guern). Le grand cycle révolutionnaire, Les Mémoires d'un médecin, doivent être lus comme l'Histoire de la Révolution française de Dumas, et confrontés aux travaux de Lamartine comme de Michelet. On peut toutefois regretter ici - mais les dimensions de ce volume sont déjà considérables - le manque de comparaisons poussées entre Dumas et ses contemporains.

Une dernier ensemble de contributions fournit l'occasion de découvrir un Dumas moins connu: un amateur d'Antiquité, un voyageur infatigable, soucieux de raconter l'histoire à partir du récit de voyage, mais capable aussi d'entrer dans l'Histoire, en débarquant à Palerme en 1860 sur les traces de Garibaldi (Claude Schopp). Ce volume invite également à la lecture de romans oubliés, comme cette fiction russe intitulée Le Maître d'Armes et réédité par Michel Arrous chez Maisonneuve et Larose. Complété par une note bibliographique fort utile, Alexandre Dumas, une lecture de l'histoire, malgré son caractère hétéroclite et - il faut le dire - parfois inégal, dessine de multiples pistes de réflexion à la charnière de l'histoire et des études littéraires, et témoigne de la vitalité de la recherche dumasienne aujourd'hui. 